\title{
Paisaje y políticas públicas en México. Una relación por resolver
}

\section{Landscape and public politics in Mexico. A relationship to be resolved}

\author{
Martín Manuel Checa-Artasu*
}

Citar este artículo como: Checa-Artasu, M. M. (2018). Paisaje y políticas públicas en México. Revista Nodo, 13(25), pp. 65-77

\section{Resumen}

El paisaje está olvidado en las políticas ambientales y territoriales de México y tiene además, una muy insuficiente consideración en el marco jurídico. A pesar de ello, desde la sociedad civil y las universidades se está realizando un notable trabajo para revertir esta pobre consideración del paisaje como herramienta de gestión territorial y ambiental y potenciando su correcta implementación en las políticas públicas mexicanas.

Palabras clave: México, Paisaje, Política pública, Universidad, Leyes.

\section{Abstract}

The landscape has been forgotten about in the environmental and territorial politics of Mexico. Furthermore, it has a very insufficient consideration in the legal framework. Despite this, civil society and universities are doing a remarkable job to reverse this poor consideration of the landscape as a territorial and environmental management tool and promoting its correct implementation in Mexican public politics.
Keywords: Mexico, Landscape, Public politic, University, Laws.

\section{Introducción}

El uso del paisaje como herramienta de análisis y de gestión en el desarrollo de políticas públicas territoriales y ambientales es muy escaso en México. Ello es resultado del pobre desarrollo de una cultura del paisaje en México desde el último cuarto del siglo XIX a la fecha (Checa, 2014). Ello ha conllevado una exigua presencia del paisaje dentro del marco jurídico mexicano y, por tanto, una escasa implementación del uso del paisaje como elemento de gestión, de ordenamiento y de protección en determinadas políticas públicas relativas al territorio y al medio ambiente. Una escasez que una vez analizada, revela un lento surgimiento de una preocupación por los diferentes problemas que lo territorial y lo ambiental están generando en el país. Una preocupación que ha sido activada y potenciada, en los últimos años desde la academia y la sociedad civil. El texto que presentamos quiere mostrar las cuatro principales líneas de actuación en relación a la actual situación del paisaje en

Fecha de recibo: 05 de julio de 2018 • Fecha de aceptación: 04 de septiembre de 2018

\footnotetext{
* Profesor-investigador de la Universidad Autónoma Metrepolitana, UAM, México. Correo electrónico: martinchecaartasu@ gmail.com
} 
México con respecto a su inserción en algunas políticas públicas concretas: la situación jurídica o legal; su presencia en ciertas acciones de política pública, la situación de la sociedad civil mexicana respecto al paisaje y lo propio, con respecto a las universidades. Nos centraremos en especial, en los cambios y novedades más recientes. Mismas que hacen intuir un futuro más halagüeño para la consideración y la valorización del paisaje en el marco de las políticas públicas mexicanas.

\section{El paisaje en la legislación mexicana. Escaso, puntual y sin objetivación}

En el grueso corpus de leyes mexicanas la presencia del paisaje es irrisoria. En ninguna ley, a la fecha, se presenta una construcción objetiva jurídicamente del mismo. Es decir, no hay norma o ley que lo defina como sujeto jurídico con cualesquiera características (Canales; Ochoa, 2009). De hecho, apenas se menciona al paisaje y cuando se hace, actúa como sinónimo de otros conceptos tanto en las normas apegadas a la protección al ambiente y del patrimonio como en las relacionadas con la gestión de los recursos naturales y el ordenamiento del territorio o la proyección de lo urbano (Aguilar Bellamy, 2006, p. 8).
Las causas de ello pudieran ser diversas: En primer término, la dificultad intrínseca que plantea la juridificación del paisaje y que no podemos soslayar. Se trata de un bien y elemento percibido y usado por el común de la población sobre el que subyace el derecho a la propiedad (lo que se percibe es de alguien). Es difícil por su polivalencia semántica y conceptual de incardinar en un área específica (derecho ambiental, territorial, administrativo, entre otros) y es un concepto emanado de la percepción humana con figuración en la realidad compleja (Martín-Retortillo, 1973, 1978).

En segundo término, y más específicamente centrándonos en México, siguiendo los planteamientos de A. Berque (2000) hay una escasa cultura del paisaje, como revela el olvido histórico de las miradas al paisaje del mundo indígena mexicano; el escaso desarrollo de la pintura del paisaje, que además requiere de estudios que la den a conocer a cabalidad y la poca presencia en general del paisaje en la literatura, ya sea decimonónica, como del siglo XX o contemporánea. Aspecto éste que al igual que en la pintura requiere de análisis y estudios hoy escasísimos. Similar postura sería si habláramos de fotografía, de cine o de otras artes plásticas (Checa, 2014, pp. 392 y ss.). En México, faltan estudios que recuperen la cultura del paisaje que sí existe y que por diversas circunstancias ha quedado olvidada y es desconocida.

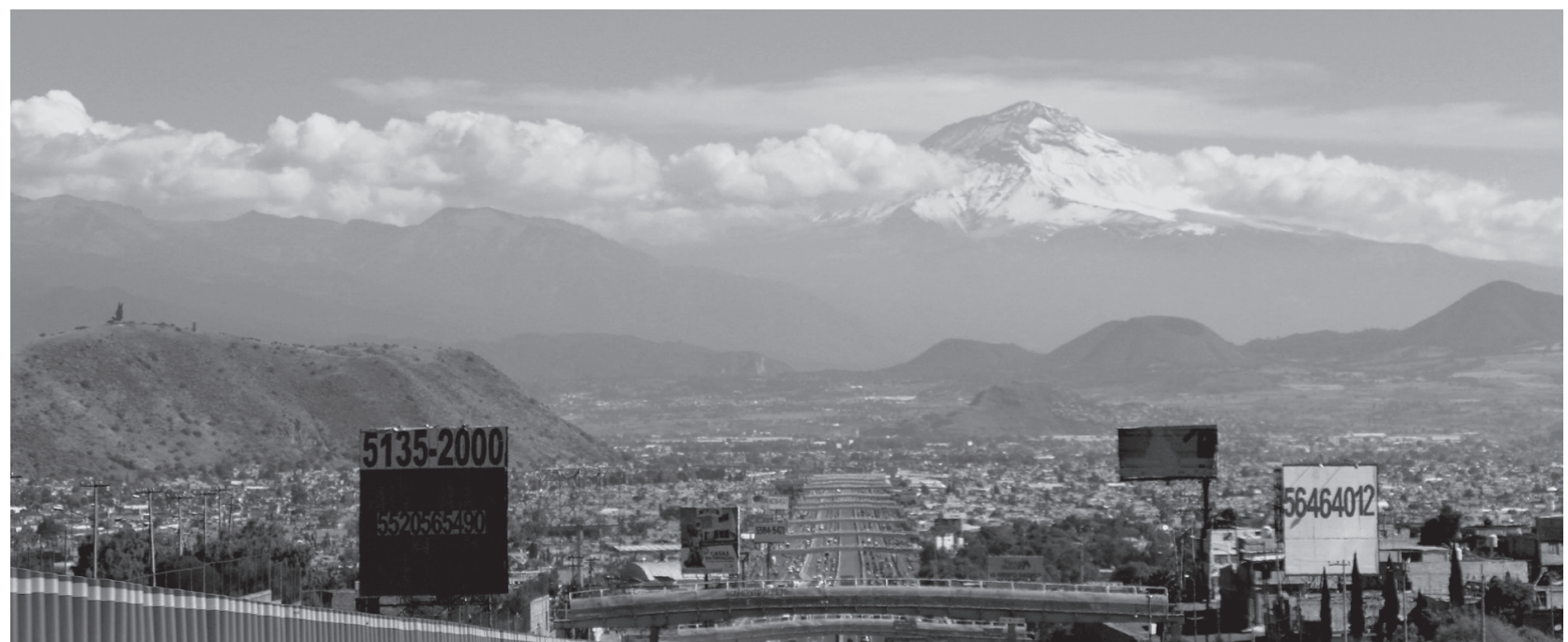

Figura 1. Paisaje urbano. Fuente: propia 
A pesar de ello, hay una consideración que se antoja más determinante para explicar la muy escasa presencia del tema del paisaje en las leyes mexicanas. Como es de sobra conocido, el territorio mexicano ha estado sujeto desde la segunda mitad del siglo XIX, y en especial a lo largo del siglo $\mathrm{XX}$, a intensos procesos de explotación de los recursos naturales; a significativas apropiaciones de territorios por parte de las élites económicas nacionales y extranjeras; a extensos repartos de tierras de carácter social, el ejido, a partir de la Revolución Mexicana y a planes de recomposición de espacios en aras de una programación gubernamental que buscaba el desarrollo económico, unilateral y homogéneo, pero marcado por el Estado en los años centrales del siglo XX, los del llamado milagro mexicano. Sorprendentemente, todo ello ha inhibido la existencia de espacios propiedad del Estado, misma que hoy es muy escasa, apenas supone el $0.28 \%$ del territorio del país (Quadri, 2016; Elizondo, 2018). A ello, se debe sumar, la competencia por recursos como los bosques, el agua, las costas, las tierras o el subsuelo, entre distintos grupos hegemónicos a partir de la asunción de las políticas neoliberales en la década de los ochenta del siglo XX. Estos grupos han hecho valer sus intereses particulares en aras del bien común. En especial, socavando los derechos humanos de numerosas comunidades indígenas y campesinas y creando hoy, numerosos conflictos socioambientales, cada vez mejor documentados y visibilizados (Paz y Risdell, 2014). En conclusión, se puede argumentar que la necesidad de desarrollo nacional en un primer momento, asociado al dominio de los recursos naturales y de territorios, en un segundo momento, han impedido que el paisaje, así como, el territorio, se haya considerado desde otra perspectiva que no sea la de la intervención invasiva y depredadora. Ello ha hecho que el medio ambiente se haya deteriorado hasta extremos increíbles. Y por supuesto, ha invisibilizado el potencial del paisaje como catalizador y organizador geográfico y ambiental.

A nuestro entender, todo ello explicaría que sean muy pocas las normas que al menos, mencionan alguna consideración respecto a la salvaguarda o valoración del paisaje tanto en el marco de la protección y gestión ambiental como en el del ordenamiento territorial (Checa, 2014). Un ejemplo de lo que mencionamos es la Ley general del equilibrio ecológico y la protección al ambiente (LGEEPA) elaborada en 1988 y modificada en 1996 y 2006 donde el término paisaje, únicamente se menciona dos veces,- ambas en el inciso segundo del artículo 47 bis,- considerando a éste como un elemento a no deteriorar sin especificar hasta qué grado de deterioro se puede alcanzar dentro de un área nacional protegida. Otro ejemplo de la indefinición jurídica del paisaje es la Ley para el Desarrollo Forestal Sustentable, aprobada en 2003. En ésta, el término paisaje se menciona dos veces, en el inciso 39 del artículo 7, donde el paisaje se enmarca como un posible servicio ambiental y en el artículo 100 donde el paisaje es una función ambiental que se puede poner en riesgo por algún tipo de aprovechamiento forestal invasivo. La indefinición de la norma en cuanto al objeto, el paisaje, y también, sobre el valor y acciones que sobre éste se hagan es clara. ¿Cómo se mide y que es el paisaje? La norma sencillamente no lo explicita. Mención aparte merece, la Ley Federal sobre Monumentos y Zonas Arqueológicas, Artísticas e Históricas, creada en 1972, misma que ha tenido que adaptarse, también de forma ambigua a la par que constreñida, a los cambios que tanto en la conceptualización,- cada vez hay más elementos susceptibles de ser patrimonio,- como en la cada vez mayor extensión territorial del patrimonio en México. La misma ni menciona, ni considera ni el paisaje ni las atribuciones contemporáneas que se hacen al mismo (paisaje cultural, industrial, religioso, entre otros.). Así, todo y una ley desfasada y la flexibilización creciente del concepto de patrimonio, México tiene en la lista de patrimonio de la humanidad algunos elementos patrimoniales donde el paisaje está presente: el paisaje del agave y las antiguas instalaciones industriales de Tequila en Jalisco, el Camino Real de Tierra Adentro, la reserva natural de Sian Ka'an en Quintana Roo y la Reserva de la Biosfera 
de la Mariposa Monarca en Michoacán. De igual forma, se incluyó en 2009 en la lista Representativa del Patrimonio Cultural Inmaterial de la Humanidad: El entorno y el paisaje de la Peña de Bernal como lugar de memoria y tradiciones vivas de los otomí-chichimecas de Tolimán. Estos ejemplos son una muestra, a nuestro entender, del uso de las convenciones internacionales en materia de protección de patrimonio para superar las limitaciones de las leyes nacionales, en especial, la relativa a la protección del patrimonio, que aún hoy, es excesivamente objetual y significativamente aterritorial.

Por debajo de la legislación federal, en México se localiza la propia de cada uno de los 32 estados del país. En relación a la mención y uso del paisaje, la situación pareciera algo mejor con respecto al paisaje, pues existen, al menos en tres estados: Tamaulipas, Querétaro y Estado de México, normas que contienen el paisaje como elemento a considerar en la conformación de un área natural protegida, recogiendo los criterios de la Unión Internacional de la conservación de la naturaleza (IUCN) en cuanto a las seis categorías de áreas naturales a considerar y atendiendo a la quinta que reconoce los paisajes terrestres y marinos. El caso, quizás más significativo es el del Código para la Biodiversidad del Estado de México que conceptualiza la categoría de paisajes protegidos como susceptibles de considerarse como áreas naturales protegidas, superando así, lo estipulado en cualquier ley federal. Un caso similar es el de la Ley de Protección Ambiental para el Desarrollo Sustentable del Estado de Querétaro quien explícitamente en su artículo 80 considera los paisajes protegidos como un área natural protegida con una serie de características. De ese modo, pareciera alinearse o facilitar respecto a la declaratoria de patrimonio intangible de la humanidad del entorno de la Peña Bernal, obtenida el mismo año, en julio de 2009, en que se publicó la ley de este Estado. Un planteamiento diferente es el de la Ley de Protección Ambiental Para El Desarrollo Sustentable del Estado de Tamaulipas que establece la categoría de paisaje natural considerando varios elementos naturales que, además, unan dos áreas naturales protegidas. Sin embargo, estas leyes son la excepción, tanto en su construcción jurídica como en su posible aplicación. La mayoría de las leyes estatales que están relacionadas con la protección y el ordenamiento ecológico consideran el paisaje desde la necesidad de protegerlo de la contaminación visual, sin llegar a decir ni que es, ni que elementos la componen, ni cómo se analiza. Se adopta, además, una asimilación entre valor escénico y paisaje en varias leyes, dejando de lado, cualquier otra característica analítica que contemple la gestión de lo territorial y lo ambiental y la promoción del desarrollo local. En este punto, se puede decir sin ambages, que la visión del paisaje en no pocas leyes estatales es excesivamente decimonónica y esteticista, alejada de la teoría contemporánea del paisaje que ya se ve en las leyes de otros países.

Recientemente, a este panorama legal bastante desordenado y confuso debemos añadir un proyecto de decreto que modificaría una serie de artículos de Ley General del Equilibrio Ecológico y la Protección del Ambiente (LGEEPA) arriba referida. Se trata de una modificación que introduciría la categoría de paisajes bioculturales como un nuevo tipo de área natural protegida (Bezaury et al., 2015). Ésta fue presentada en la Cámara de Diputados en octubre de 2016 por el Partido Verde Ecologista de México y aún está en la fase de tramitación parlamentaria, a la espera de la resolución por parte del Senado mexicano. Se trata de un cambio cabildeado por organizaciones no gubernamentales mexicanas dedicadas a la conservación de la biodiversidad desde 2011. Éste busca aumentar la superficie natural a conservar del país, actualmente $13 \%$, y elevarla al $17 \%$, siguiendo las metas de Aichi suscritas por México en 2010. Hay que decir que este cambio normativo viene a cubrir una serie de obligaciones jurídicas derivadas de acuerdos internacionales suscritos por México (protocolo de Nagoya y Metas de Aichi).

Para dicha modificación de esta ley se toma la definición de paisaje de la Unión Internacional de la Conservación de la Naturaleza (IUCN) como 


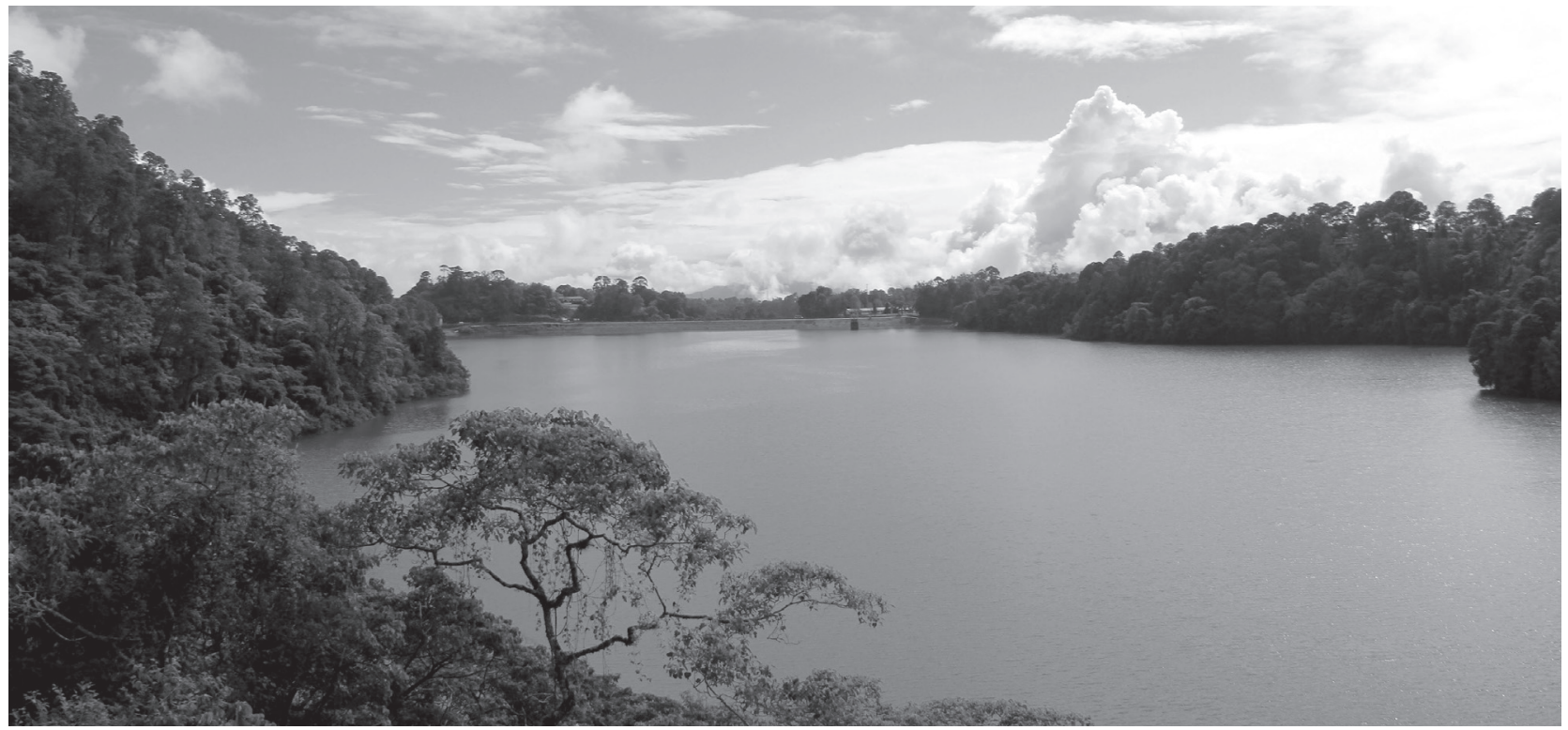

Figura 2. Paisaje natural. Fuente: propia

eje medular. Misma que como hemos relatado unas líneas más atrás, ya se había considerado en algunas normas estatales. Además de todo ello, dicho cambio se adapta a la realidad territorial, conservacionista y jurídica mexicana la experiencia francesa de los Parques naturales regionales: creando la figura de los paisajes bioculturales como áreas naturales protegidas (Bezaury; Rojas, 2012). Asimismo, conviene decir que la modificación legal que se propone y su resultado, los ya citados: paisajes bioculturales, se alinean a los presupuestos teóricos que relacionan la alta presencia de biodiversidad con altas concentraciones de diversidad cultural, específicamente indígena (Toledo, 2001). Esta modificación tiene, además, una gran trascendencia para la valorización del paisaje en México ya que lo situaría por primera vez como un sujeto jurídico de protección y gestión y sobre todo, objetivado jurídicamente. La propuesta que en apariencia es buena, espera su aprobación para poder ser implementada y descubrir o no, sus bondades. Por lo pronto, antes de su aprobación ya ha hay una iniciativa pionera, desarrollada por la Comisión Nacional de Áreas Naturales Protegidas (CONANP) y la ONG Espacios Naturales y Desarrollo Sustentable, A.C.
(ENDESU) y patrocinada por la Agencia Francesa para el Desarrollo, denominada: Preservación de la biodiversidad y de los ecosistemas del corredor Ameca - Manantlán en Jalisco ${ }^{1}$, quien tomando el concepto de paisajes bioculturales y en especial, la gestión territorial asociada este concepto, está desarrollando el mismo proceso que se prevé será reconocido por la modificación de la LGEEPA a aprobar (Bessy et al., 2016).

\section{Centelleos de paisaje en las políticas públicas ambientales y territoriales de México}

Hablar de paisaje y políticas públicas no está exento de complejidades. Como ya hemos señalado, el concepto de paisaje es polivalente y a ratos, impreciso por la variedad de actores que pueden intervenir en su interpretación. Ello, sin embargo, no ha impedido que, a partir de la construcción de un aparato legal, el paisaje haya penetrado en

1 Sobre este proyecto se debe consultar: http://endesu. org.mx/coordinacio-n-institucional/proteccion-de-la-biodiversidad-del-corredor-ameca-manantlan/\#.XAnLRdtKga8 
las políticas públicas de no pocos países y desde distintas perspectivas (Serrano, 2007, p. 110). En México, la presencia del paisaje en las políticas públicas es ciertamente escasa, aunque ya se observan algunos centelleos que hacen pensar que en cuanto se active la consideración legal del paisaje, este ganará posiciones en marcos concretos de políticas públicas.

Hemos mencionado en unas líneas más arriba, que la Ley General del Equilibrio Ecológico y la Protección del Ambiente (LGEEPA) no menciona apenas nada respecto al paisaje. Sin embargo, curiosamente es desde esa misma ley donde el uso del paisaje como elemento de ordenamiento puede tener visos de aplicación en México. Por un lado, a partir de los llamados ordenamientos ecológicos, un mecanismo de cumplimiento a nivel estatal y municipal que requiere de una regionalización ecológica de un área administrativa (Rosete, 2006, p. 32; Azuela, 2006). Este ejercicio se puede plantear con un enfoque desde el paisaje, a través de un análisis de unidades del paisaje, como ya se ha hecho en algún caso (Arreola, 2008). Hay que añadir que ese análisis depende de unos criterios de estudio, capas de contenidos abióticos, bióticos y antrópicos, fortalecidos por el uso de cartografía digital y sistemas de información geográfica. Criterios que surgen de una larga tradición de investigaciones proveniente de la ecología del paisaje.

Por otro lado, a partir de la LGEEPA se ha incentivado el desarrollo de los ordenamientos ecológicos comunitarios, mismos que suscitan una apropiación del territorio por parte de comunidades agrarias indígenas y de paso, un inicio de planeación ambiental y ordenamiento territorial para importantes zonas del país (Anta et al., 2008; Negrete y Bocco, 2003). El ordenamiento ecológico comunitario incorpora el paisaje como un componente más y de forma indirecta, éste se incorpora a los principios etnoecológicos que deben considerarse para el desarrollo sustentable de esas comunidades (Toledo, 1996).

Otro punto que hay que mencionar en este apartado relativo a las políticas públicas es el deseo de utilizar el manejo integrado del paisaje como herramienta de gestión en las áreas naturales protegidas de México. Una gestión que hay que decirlo, ha sido ciertamente disímil e inconstante. Lo dicho se constata en el hecho que dependencias federales como la Comisión Nacional de Áreas Naturales Protegidas (CONANP) junto con la Secretaría de Medio Ambiente y Recursos Naturales (SEMARNAT) plantearon en el periodo de gobierno (2012-2018) la propuesta de gestión denominada: Estrategia hacia 2040, donde se presentaba como un eje sustantivo de la misma, el manejo integrado del paisaje (CONANP, 2014). Entendido éste como una herramienta para integrar tanto la conservación de la biodiversidad como el fomento al desarrollo de realidades sociales y económicas que se dan en las áreas naturales protegidas. Vale la pena añadir que se trataba de una propuesta de gestión donde el paisaje se pretendía analizar desde los presupuestos de la ecología del paisaje. Se entendía éste como un sinónimo de territorio, como un recurso, como un mosaico de posibles servicios ecosistémicos y como potenciador de desarrollo sostenible. Sin embargo, este planteamiento parecía olvidar algunos aspectos más culturales como la identidad emanada de los paisajes, su proceso evolutivo, su historicidad, el papel de la percepción en su análisis y la diversidad de metodologías para el uso y análisis del paisaje provenientes de otras disciplinas. Esto, sumado al reciente cambio de gobierno a nivel federal en México pareciera condenar la mencionada estrategia a ser pospuesta o olvidada.

Finalmente, hay que comentar que desde la academia se han realizado algunos análisis que bien pudieran transformarse en el medio plazo en políticas públicas. Todos basados en estudios desde la ecología del paisaje fundados en criterios físico-ambientales e instrumentalizados a través de la construcción de unidades del paisaje. Entre estos vale la pena señalar los relativos al manejo de cuencas hidrográficas, por ejemplo, la cuenca Lerma-Chapala o los que hacen referencia a procesos de restauración ambiental (Cotler et al., 2005). 


\section{El paisaje lentamente fortalecido por la sociedad civil}

En México, durante la última década se han fortalecido y han surgido asociaciones que entre sus principales objetivos está el estudio, análisis y difusión de la importancia del paisaje como herramienta de gestión territorial y de compromiso con el ambiente. Dichas entidades, son una muestra de la rica sociedad civil mexicana, organizan congresos, conferencias, bienales y editan, de tanto en tanto, publicaciones. Entre éstas destacan: la Sociedad de Arquitectos Paisajistas de México (SAPM) fundada en 1972 por un grupo de jóvenes arquitectos del paisaje ${ }^{2}$; la Red Mexicana de estudios sobre paisajes patrimoniales (REMEPP) creada en 2014 al influjo de la realización de las primeras jornadas del mismo nombre en Puebla; la Academia Mexicana del paisaje (ACAMPA) iniciada en $2003^{3}$, aunque legalizada en 2008, con la participación de arquitectos y biólogos de la Universidad Nacional Autónoma de México y la Universidad de Guadalajara y la Fundación de paisajes culturales creada en 2012 por varias arquitectas paisajistas con la clara vocación de dar a conocer paisajes desde la difusión ${ }^{4}$.

Han sido estas asociaciones las que han vehiculado la redacción de documentos programáticos que alertaban de la importancia del paisaje en una sociedad como la mexicana, convulsionada por la violencia y la corrupción y donde los derechos humanos están en franco retroceso. Hay dos ejemplos de ello. El primero, por parte de la Sociedad de Arquitectos Paisajistas de México (SAPM), en noviembre de 2010, publicó la Carta Mexicana del paisaje, un ejercicio de mínimos que pretendía ser la base de una futura norma relacionada con la protección y gestión del paisaje en México. Este

2 Sobre la Sociedad de Arquitectos Paisajistas de México, consultar: https://www.sapm.com.mx/

3 Sobre esta asociación hay que consultar: https://www. acampa.land/

$4 \quad$ Ver el blog: https://paisajesculturales.mx/ documento, sin valor normativo, quería llamar la atención de la riqueza de los paisajes mexicanos y de la necesidad de conservarlos y de integrarlos en los ordenamientos urbanos y territoriales, entendiendo su riqueza ecológica pero también educativa, económica, cultural y social. Se trata de una propuesta, que coincide plenamente con otras iniciativas similares en Latinoamérica (Argentina, Venezuela, Ecuador, Perú, Bolivia, Uruguay, Colombia, Costa Rica, Brasil y Chile), que en no pocos casos, con la mediación de la Federación Internacional de arquitectos paisajistas dieron pie, en 2012, a la declaración de la Iniciativa Latinoamericana del paisaje (LALI). Elemento programático fundamental para entender el fortalecimiento del paisaje como concepto y como elemento de política pública en el continente. El segundo ejemplo se da en 2014, en otra entidad, Red Mexicana de Estudios sobre Paisajes Patrimoniales (REMEPP) presentaba la Carta de Puebla de protección de paisajes patrimoniales 5 . Una declaración cívica sobre el valor y la necesidad de difundir y preservar este tipo de paisaje, donde lo patrimonial estructura los mismos y se localizan a lo largo y ancho del país.

Otro elemento que merece ser reseñado es la defensa de un paisaje por parte de la sociedad civil. Sin ser todavía muy numerosos, en los últimos quince años han aparecido en México diversos colectivos de ciudadanos que tienen en común la protección y defensa de un determinado patrimonio cultural. Ese patrimonio muchas veces se incardina en un contexto urbano, en otras, las menos, es de carácter natural y se vincula a la defensa de un paisaje determinado. Por lo general, no se busca simplemente la defensa de un único edificio, su acción pasa por la valorización de un conjunto que conforma un paisaje, ya sea urbano, ya sea natural, que recoge la memoria y la identidad de los habitantes de ese entorno. Dos casos merecen ser reseñados en este sentido: el de la

5 Ver la página de Facebook de esta entidad: https://www. facebook.com/Red-Mexicana-de-Estudios-sobre-PaisajesPatrimoniales-1749369228653842/ 


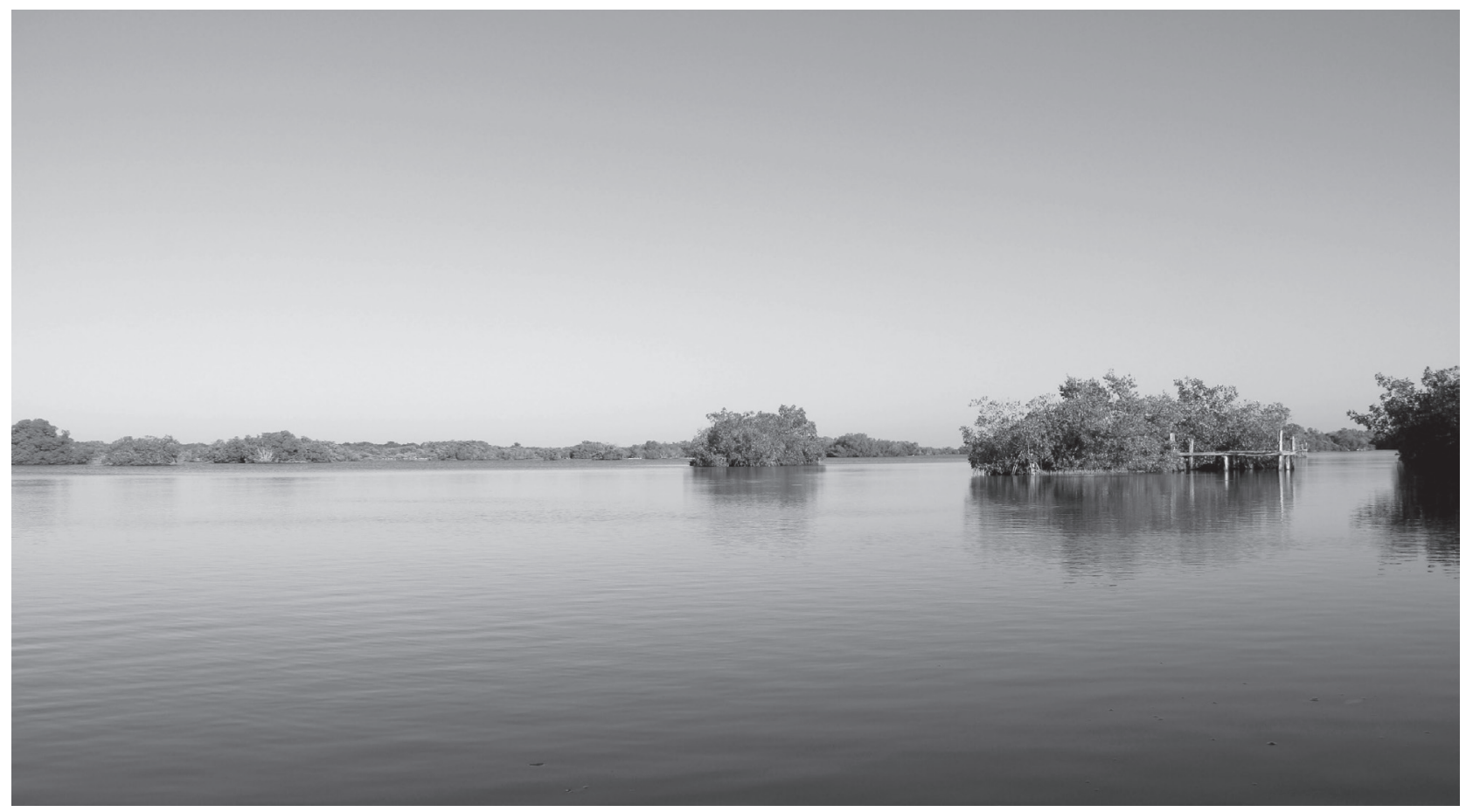

Figura 3. Paisaje de agua. Fuente: propia

defensa de La Bufa y los Picachos en Guanajuato ciudad y la defensa del paisaje vitivinícola del Valle de Guadalupe en Enseñada, Baja California (Checa, 2017, pp. 48 y s.) En ambos casos, la ciudadanía organizada consiguió que se hiciera una consulta ciudadana o negoció en términos políticos para evitar un desarrollo inmobiliario muy agresivo que iba a descomponer un paisaje considerado como propio en ambos casos.

\section{Los pasos del paisaje en la universidad mexicana}

El estudio y análisis del paisaje desde la universidad mexicana se ha incrementado y consolidado desde la última década. Varios elementos parecen constatarlo. El primero, desde la academia en colaboración con las entidades antes señaladas y a veces, con la participación de museos y centros de investigación diversos, se ha organizado congresos y ciclos de conferencias. Recientemente, además, han aparecido estudios de posgrado en varias universidades del país, con una mirada concreta hacía paisaje desde diversas disciplinas. Estos se suman a toda la serie de otros posgrados de disciplinas como la arquitectura, la geografía o los estudios ambientales donde desde hace tiempo se presentan tesis, algunas novedosas en cuanto a la forma de analizar el paisaje. En este sentido es especialmente, encomiable la labor realizada por el Instituto de Geografía y en el Departamento de Arquitectura del Paisaje de la Universidad Nacional Autónoma de México (UNAM) en la Ciudad de México ${ }^{6}$. Así como, los trabajos del Centro de Investigaciones en Geografía Ambiental y su homónimo en ecología, de la UNAM, ambos en Morelia, quienes desde la perspectiva de la ecología del paisaje han desarrollado diversas tesis y proyectos. De igual forma, hay que mencionar, el papel de algunos investigadores adscritos al Colegio de Michoacán, al Colegio de la Frontera Sur, al Instituto de Ecología, al CIESAS Occidente, a la Benemérita Universidad Autónoma de Puebla, a la licenciatura de geografía de la Universidad

6 Sobre esta institución consultar: http://arquitectura. unam.mx/arquitectura-de-paisaje.html 
Autónoma Metropolitana, Unidad Iztapalapa y del Área de investigación en Arquitectura del Paisaje del Departamento de Arquitectura y Diseño de la Universidad Autónoma Metropolitana, Unidad Azcapotzalco que han realizado no pocos análisis, algunos en curso, que se enmarcan dentro de disciplinas como la geografía, la historia, la ecología o la arquitectura ${ }^{7}$. Análisis que van de la mano al desarrollo del posgrado en Diseño, Planificación y Conservación de Paisajes y Jardines de la Universidad Autónoma Metropolitana, Unidad Azcapotzalco ${ }^{8}$.

Un apartado especial merece la arquitectura del paisaje mexicana ya que en los últimos 25 años ha venido haciendo distintas propuestas, tanto desde la docencia como desde la acción profesional. En distintas universidades del país se han articulado cursos, maestrías, seminarios, bienales y concursos que ponían en consonancia formación y acción práctica y real. De igual forma, a lo largo de esos años han aparecido estudios de arquitectura con una cierta especialización en el paisaje y ha habido la consolidación, incluso a nivel internacional, de algunos arquitectos (Lutteroth y Martínez, 2006). Ejemplos de ello son: Mario Schjetman, cuya obra ha adquirido gran reconocimiento internacional; la arquitecta nuevoleonesa Claudia Harari ${ }^{9}$, el arquitecto holandés afincado en el país: Kees Van Rooij, socio desde 1994 de KVR Arquitectura del Paisaje $^{10}$, los numerosos trabajos de la arquitecta mexicana Desirée Martínez Uriarte; los proyectos de los estudios de arquitectura: Paisaje Mexicano de la arquitecta Esperanza Viramontes ${ }^{11}$, de LAAP Landscape, de Entorno, arquitectura del paisaje,

7 Sobre este grupo de investigación se ha de consultar: http://www.cyad.azc.uam.mx/arquitecturadelpaisaje.php

8 Sobre ese posgrado consultar: http://cyadposgrados. azc.uam.mx/paisajes_jardines.html

9 Sobre este estudio hay que consultar: http://www.hararilandscape.mx/

10 La actividad de este profesional es consultable en: http:// www.kvr-paisaje.com/

11 Ver: http://www.paisajemexicano.com.mx/ en Puebla, Ecotono Urbano en Monterrey ${ }^{12}$, Muray Paisajistas o Buro Verde arquitectos.

En cuanto a las publicaciones, baste decir que en los últimos quince años se han publicado paulatinamente diversas monografías y libros coordinados en relación con el paisaje que han puesto la temática en la palestra y el debate intelectual, aun cuando falta mayores asideros con la sociedad y los decisores políticos (García, 2002; Fernández Christlieb y García Zambrano, 2006; Thiébaut et al., 2008; Hernández López, 2013; Checa et al., 2014, Castellanos, 2015; Larrucea, 2016; Checa y Sunyer, 2017; Castellanos et al., 2017). De igual forma, se han publicado una significativa cantidad de artículos con aproximaciones al paisaje desde diversas disciplinas y con múltiples características. Por un lado, parecen dominar, con una notoria bibliografía en español y en inglés, los análisis surgidos desde la ecología del paisaje, en especial, los vinculados al estudio de especies tanto vegetales como animales, la dispersión y la movilidad de estas y la fragmentación de paisajes, entendidos como unidades con características unitarias. Tanto es así, que pareciera han trazado una línea de análisis del paisaje concreta para disciplinas como la geografía o las ciencias ambientales o la biología (García Romero, 2002). Por otro lado, son más escasos, aquellos que estudian una realidad geográfica valorando la acción del hombre y su actividad como conformador de paisajes (Boehm, 2001; Hernández López, 2013; Mollá, 2010; Porter-Bolland et al., 2008; Thiébaut, 2008, 2011a ,2011b, 2013). Igualmente, son excepcionales los trabajos que ahondan en los paisajes considerando su componente histórico y por tanto, evolutivo (Garza, 2000; Fernández Christlieb; García Zambrano, 2006). Finalmente, cabe añadir que se detecta aún pocos trabajos con el interés de profundizar teóricamente en la concepción del paisaje y en sus posibles especificidades en México. De ello resulta, que apenas se localicen unos pocos trabajos en este rubro (Aguilar Bellamy, 2006;

12 Ver: http://www.ecotonourbano.com 
Urquijoy Bocco, 2011; Larrucea, 2016; ChecaArtasu, 2014, 2017, 2018; Sunyer, 2017).

Finalmente, hay que mencionar que desde la academia se están generando métodos de análisis del paisaje con el uso de diversas metodologías. En algunos casos, se promueven métodos para automatizar la construcción de unidades del paisaje y vincularlas a los procesos de ordenamiento ecológico que se están dando en el país (Priego et al, 2008). Procesos donde, por ejemplo, en la determinación de unidades de gestión ambiental se contempla la continuidad de los paisajes como parte integral de las mismas. Todo y los excesos fisicalistas de esta mirada al paisaje, creemos que se trata de una oportunidad en construcción para una mayor consideración del paisaje en el marco de las políticas públicas de México, tal como hemos señalado más arriba.

\section{Conclusiones}

A lolargo de estas líneas hemos podido documentar la situación del paisaje con respecto a su posible inserción en las políticas públicas de México. Por un lado, detectamos una casi nula presencia en la legislación, con apenas menciones en algunas leyes relacionadas con diversas cuestiones ambientales. Un hecho que pudiera cambiar próximamente, debido a la creciente actividad desde la academia en relación a esa cuestión y el creciente interés sobre el paisaje de ciertos sectores de la sociedad. Hemos presentado, igualmente, un repaso muy somero a las políticas públicas en cuanto al ordenamiento territorial y ecológico y también, en relación a la gestión de las áreas naturales protegidas, parecieran darle alguna oportunidad al uso del paisaje desde las concepciones propias de la ecología del paisaje. Lamentablemente, todo ello es incipiente dada la franca debilidad jurídica del paisaje en México.

Un panorama mucho más esperanzador parece detectarse al mirar las iniciativas de la sociedad civil mexicana en cuanto al paisaje. Se observa la aparición de asociaciones y el desarrollo de congresos y jornadas, con especial predicamento en los últimos cinco años. Algo similar se observa analizando las actividades hechas en las universidades mexicanas en la última década. Por un lado, han aparecido posgrados desde diversas disciplinas y también, algunos específicos donde el paisaje es el elemento medular. En cuanto a las publicaciones ha habido un crecimiento de las mismas, especialmente de monografías y libros coordinados que ha servido para sentar las bases para indagar sobre el paisaje, ya no sólo desde el estudio de caso concreto y focalizado en un espacio geográfico sino para tratarlo en términos teóricos, conceptuales y de gestión. De todo lo dicho en estas líneas se puede desprender una conclusión significativa, hay un creciente interés por y en el paisaje, mismo que se está construyendo y discutiendo desde diversos frentes, quizás todavía desconectados entre sí. Una construcción incipiente que requiere redoblar esfuerzos para incardinarla en los procesos donde los agentes políticos toman postura ante problemáticas que desde la sociedad son sujetas al escrutinio de la opinión pública. Este paso es prioritario para que finalmente, en México se cuenten con políticas públicas donde el paisaje sea un elemento de gestión que redunde en un mejor manejo del territorio y el medio ambiente.

\section{Referencias bibliográficas}

Aguilar Bellamy, A. (2006). Algunas consideraciones teóricas en torno al paisaje como ámbito de intervención institucional. Gaceta Ecológica, núm. 79, pp. 68-82.

Anta Fonseca, S., Arreola Muñoz, A.V., González Ortiz, M.A.y Acosta González, J. (comps.) (2008). Ordenamiento territorial comunitario, Ciudad de México: Secretaría de Medio Ambiente y Recursos Naturales.

Arreola, A. (2008). Ordenamiento territorial del municipio Calakmul, Campeche, un estudio enfocado a las funciones del paisaje. Ciudad de México: Comisión Nacional de Áreas naturales protegidas. 
Azuela, Antonio (coord.) (2006). El ordenamiento ecológico del territorio en México: génesis y perspectivas. Ciudad de México: Secretaría de Medio Ambiente y Recursos Naturales.

Berque, Augustin (2000). Écoumène. Introduction à l'étude des milieux humains. Paris: Editions Belin.

Bessy, A., Leparquier, F., Bourbousson, C., Mercier, D. y Bahuchet, S. (2016). 3ro informe del proyecto de investigación MI SIERRA: Gobernanza y usos de la biodiversidad en la Sierra Occidental de Jalisco.Ciudad de México : Centre d'Etudes Mexicaines et Centraméricaines, CEMCA; Muséum d'Histoire Naturelle; Agence Française de Développement.

Bezaury-Creel, J., Graf-Montero, S., BarcklayBriseño, K., De la Maza-Hernández, R., Machado-Macías, J.S., Rodríguez-Martínez del Sobral, E., Rojas-González de Castilla, S. y RuízBarranco, H. (2015). Los Paisajes Bioculturales: un instrumento para el desarrollo rural y la conservación del patrimonio natural y cultural de México. Ciudad de México: Comisión Nacional de Áreas naturales protegidas.

Canales Pinacho, F., Ochoa Gómez, P. (2009). La juridificación del paisaje o de cómo convertir un criterio esencialmente estético en un bien jurídico objetivable. Diario La Ley, núm. 7183, pp. 1-34.

Bezaury-Creel, J.E. y Rojas González, S. (2012). Análisis del panorama institucional mexicano para determinar la pertinencia y factibilidad de adaptar este nuevo modelo de gestión territorial al contexto mexicano. Ciudad de México: Agencia Francesa de Desarrollo; Comisión Nacional de Áreas Naturales protegidas; The Nature Conservancy.

Boehm Schoendube, B. (2001). El lago de Chapala: su ribera norte. Un ensayo de lectura del paisaje cultural. Relaciones, núm. 85, pp. 57-85.

Castellanos Arenas, M. (2014). El Patrimonio Cultural Territorial. Paisaje, historia y gestión.
Puebla: Benemérita Universidad Autónoma de Puebla; Educación y cultura, Asesoría y promoción.

Castellanos Arenas, M., Vélez Pliego, F.y Hernández Amador, E. (eds.) (2017). Paisajes Patrimoniales. Investigación y gestión en el siglo XXI. Puebla: Instituto de Ciencias Sociales y Humanidades Alfonso Vélez Pliego, Benemérita Universidad Autónoma de Puebla; Editora Educación y Cultura. Asesoría y Promoción

Checa-Artasu, M., García Chiang, A., Soto Villagrán, P. y Sunyer Martín, P. (coords.) (2014). Paisaje y territorio. Articulaciones teóricas y empíricas. Ciudad de México: Universidad Autónoma Metropolitana, Unidad Iztapalapa; Editorial Tirant Lo Blanch.

Checa-Artasu, M. (2018). De la percepción a la consideración como derecho. El largo camino para el paisaje en México. En: A. Alonso Navarrete; F. Martínez Sánchez (coords.). Arte, Historia y Cultura. Nuevas aproximaciones al conocimiento del paisaje (pp. 159-180). Ciudad de México: Universidad Autónoma Metropolitana, Unidad Azcapotzalco.

Checa-Artasu, M. y Sunyer Martín, P. (coords.) (2017). Paisaje: métodos de análisis y reflexiones. Ciudad de México: Universidad Autónoma Metropolitana, Unidad Iztapalapa; Editorial del Lirio.

Checa-Artasu, M. (2017). En defensa del derecho al paisaje. Algunos ejemplos en México. En M.M. Checa-Artasu.; P.Sunyer Martín (coords.). Paisaje: métodos de análisis y reflexiones (pp. 45-73). Ciudad de México: Universidad Autónoma Metropolitana, Unidad Iztapalapa; Editorial del Lirio.

Checa-Artasu, M. (2014). Oportunidades y carencias para una cultura del paisaje en México. Algunas notas. En M. Checa-Artasu.; A. García Chiang; P. Soto Villagrán; P.Sunyer Martín (coords.). Paisaje y territorio. Articulaciones teóricas y empíricas (pp. 389-423). Ciudad de México: Universidad Autónoma Metropolitana, Unidad Iztapalapa ; Editorial Tirant Lo Blanch. 
CONANP (2014). Estrategia hacia 2040: una orientación para la conservación de las áreas naturales protegidas de México. Ciudad de México: Comisión Nacional de Áreas Naturales Protegidas, Secretaría de Medio Ambiente y Recursos Naturales.

Cotler, H. y Priego, A. (2004). El análisis del paisaje como base para el manejo integrado de cuencas: el caso de la cuenca Lerma-Chapala. En H.Cotler (comp.). El manejo integral de cuencas en México: estudios y reflexiones para orientar la política ambiental (pp. 63-74). Ciudad de México: Instituto Nacional de Ecología, SEMARNAT.

Cotler H., Bocco, G. y Velásquez, A. (2005). El análisis del paisaje como base para la restauración ecológica. En: O. Sánchez. Diplomado en restauración ecológica. Ciudad de México: Instituto Nacional de Ecología, SEMARNAT, United States Fish \& Wildlife Service, Unidos para la Conservación, A. C., Centro de Investigaciones en Ecosistemas, Universidad Nacional Autónoma de México.

Elizondo Mayer-Serra, C. (2018). Constitución y territorio propiedad del Estado: dos casos polares Revista Mexicana de Sociología, núm. 80(2), pp. 353-383.

Fernández Christlieb, F. y García Zambrano, Á.J. (coords.) (2006). Territorialidad y paisaje en el altépetl del siglo XVI. México: Fondo de Cultura Económica.

García Romero, Arturo (2002). El paisaje en el ámbito de la geografía. Ciudad de México: Instituto de Geografía, Universidad Nacional Autónoma de México.

Garza Merodio, G.G. (2000). Evolución en el paisaje de la cuenca de México durante la dominación española, tesis doctoral, Facultat de Geografía e Historia, Universitat de Barcelona.

Hernández López, J. J. (2013). Paisaje y creación de valor. Las transformaciones de los paisajes culturales del agave y del tequila. Zamora: El Colegio de Michoacán, p. 349.
Larrucea Garritz, A. (2016). País y Paisaje. Dos Invenciones del Siglo XIX mexicano. Ciudad de México: Universidad Nacional Autónoma de México.

Lutteroth Alonso, L. y Martínez Uriarte, D. (Eds.) (2006). Encuentro de espacios: Arquitectura de Paisaje Mexicana. Múnich: Callwey $\mathrm{GmbH}$ \& Co; Sociedad de Arquitectos Paisajistas de México.

Martín-Retortillo Baquer, L. (1973). Problemas jurídicos de la tutela del paisaje, Revista de Administración Pública, núm. 71, pp. 423-442.

Martín-Retortillo Baquer, L. (1978). Problemas jurídicos del paisaje. Argensola: Revista de Ciencias Sociales del Instituto de Estudios Altoaragoneses, núm. 85, pp. 25-40.

Mollá Ruiz-Gómez, M. (2010). Paisajes identitarios: México. En: E. Martínez de Pisón; N. Ortega Cantero (eds.). El paisaje: valores $e$ identidades (pp.102-121). Madrid, Fundación Duques de Soria, Ediciones Universidad Autónoma de Madrid.

Negrete, G. y Bocco, G. (2003). El ordenamiento ecológico comunitario: una alternativa de planeación participativa en el contexto de la política ambiental de México Gaceta Ecológica, núm. 68, pp. 9-22.

Paz, M.F. y Risdell, N. (coords.) (2014). Conflictos, conflictividades y movilizaciones socioambientales en México. Problemas comunes, lecturas diversas. Ciudad de México: Centro Regional de Investigaciones Multidisciplinarias de la Universidad Nacional Autónoma de México; Miguel Ángel Porrua Editor.

Porter-Bolland, L., Sánchez González, M.C. y Ellis, E.A. (2008). La conformación del paisaje y el aprovechamiento de los recursos naturales por las comunidades mayas de La Montaña, Hopelchén, Campeche Investigaciones Geográficas, Boletín del Instituto de Geografía, núm. 66, pp. 52-71.

Priego, Á., Bocco, G., Mendoza, M. y Garrido, A. (2008). Propuesta para la generación semiautomatizada de unidades de paisajes. Ciudad 
de México: Secretaría de Medio Ambiente y Recursos Naturales; Instituto Nacional de Ecología; Centro de Investigaciones en Geografía Ambiental, Universidad Nacional Autónoma de México.

Quadri, G. y Quadri, P. (2016). México, un Estado sin tierra. Hacia una propiedad pública de la tierra en Áreas Naturales Protegidas. Ciudad de México: Miguel Ángel Porrúa, librero-editor.

Rosete, F. (2006). Semblanza histórica del ordenamiento ecológico territorial en México. Perspectiva institucional. Ciudad de México: Instituto Nacional de Ecología.

Sunyer Martín, P. (2017). Paisajes para todos. De la valorización del paisaje a su sensibilización. En M. Checa-Artasu; P. Sunyer Martín (coords.). Paisaje: métodos de análisis y reflexiones (pp. 21-44). Ciudad de México: Universidad Autónoma Metropolitana, Unidad Iztapalapa; Editorial del Lirio.

Thiébaut, V., García Sánchez, M. y Jiménez Izarraraz, A. (eds.) (2008). Patrimonio y paisajes culturales. Zamora: El Colegio de Michoacán.

Thiébaut, V. (2013a). Procesos rurales en México. La génesis de los paisajes de una microcuenca de Michoacán Ería. Revista cuatrimestral de Geografía, núm. 91, pp. 151-166.

Thiébaut, V. (2013b). Paisaje e identidad. El río Papaloapan, elemento funcional y simbólico de los paisajes del Sotavento Liminar. Estudios Sociales y Humanísticos, vol. XI, núm. 2, pp. 82-99.

Thiébaut, V. (2011). Paisajes identitarios en México. Análisis y valoración de paisajes de la independencia Estudios Geográficos, vol. 72, núm. 271, pp. 655-680.

Toledo, V. (1996). Principios etnoecológicos para el desarrollo sustentable de comunidades campesinas e indígenas, núm. 4. Disponible en: [http://ambiental.net/temasclave/TC04ToledoEtnoecologiaPrincipios.htm] [Consulta: 26 de abril de 2018].

Toledo., V.M. (2001). Indigenous peoples and biodiversity. En Encyclopedia of Biodiversity, vol. 3, pp. 451-463.

Urquijo, P. y Bocco, G. (2011). Los estudios de paisaje y su importancia en México, 1970-2010 Journal of Latin American Geography, núm. 10 (2), pp. 37-63. 